\title{
Thanks to JMCP Peer Reviewers, 2019
}

The Journal of Managed Care \& Specialty Pharmacy would like to thank the 308 reviewers who have evaluated manuscripts in 2018 and contributed to the high quality of articles in JMCP. Without this excellent level of reviewer involvement, $J M C P$ would not be one of the most respected journals in health care services, connecting authors and innovators to accelerate advances in managed care pharmacy.

\section{—Laura E. Happe, PharmD, MPH} Editor-in-Chief

Paul N. Abourjaily, PharmD, BCPS

Safiya Abouzaid, PharmD, MPH

Susan Abughosh, PhD

Sarah Elaine Adkins, BS, RPh, PharmD

Jeremy Adler, MD, MSc

Adewale Ajumobi, MD, MBA

Medinat S. Akinbi, PharmD

Sheila Albeke, PharmD

Zain A. Al-Jammali, PharmD

Arnie Aldridge, $\mathrm{PhD}$

James Daniel Allen, PharmD

JaeJin An, BPharm, PhD

Jennifer Andres, PharmD

Rebekah Hanson Anguiano, PharmD, BCPS

Dana Angela Drzayich Antol, MS

Hilary A. Aroke, PhD, MD, MPH, MS

Steve G. Avey, MS, RPh

Jay P. Bae, PhD

Danial E. Baker, BPharm, PharmD

Jennifer Waymier Baker, PharmD, BCPS, BCACP

Vishal Bali, PhD

Tannista Banerjee, PhD

Mitchell J. Barnett, PharmD, MS

Ramsankar Basak, PhD

Christopher Bell, MS

Brandon K. Bellows, PharmD, MS

Todd Berner, MD

Sandipan Bhattacharjee, BPharm, MS, PhD

Sarah J. Billups, PharmD

Joseph Biskupiak, PhD, MBA

Crystal Blankenship, PharmD

Christopher Blum, PharmD

Mary Ellen Bonk, PharmD

Nancy Borja-Hart, PharmD
Eric P. Borrelli, PharmD, MBA

Joel Brill, MD

Erin A. Brown, PharmD

Joshua David Brown, PharmD, PhD

Ami R. Buikema, MPH

George Bungey, BSc

Russel Burge, PhD

Elizabeth Sutton Burke, PharmD

Jonathan D. Campbell, PhD

Norman V. Carroll, PhD

Sara Carruth, PharmD

Vivien Chan, PharmD

Kevin Chang, PharmD

Jingdong Chao, $\mathrm{PhD}$

Satabdi Chatterjee, MS, PhD

Anita Chawla, PhD

Hua Chen, PhD

Judy W. Cheng, PharmD

Sumit Chhabra, BPharm, BSPharm, MS, $\mathrm{RPh}$

Lauren Chin, PharmD

Chanadda Chinthammit, BPharm, MS, PhD

Pooja Chopra, MS

Chiahung Chou, PhD

Shelley Chun, PharmD

Vickie Pon Chung, PharmD

Karen M. Clements, ScD

Daniel M. Cook, PhD

William Crown, PhD

Brian Keith Crownover, MD

Eric J. Culley, PharmD, MBA

Suellen Curkendall, $\mathrm{PhD}$

Akash Danavar, PharmD, MS, MPH, MA

Jonathan J. Darrow, SJD, JD, MBA

Ankur A. Dashputre, MS
Desola Davis, PharmD

Mary Lynn Davis-Ajami, PhD, MBA, MSN, FNP-C, RN

Devender Dhanda, PhD, MBA, MS

Marco DiBonaventura, PhD

Thomas J. Dilworth, PharmD

Qian Ding, PhD

Alex Dong, PharmD

Aaron Douglas Drovandi, MPharmPH

Michael Drummond, PhD

Emily Durden, $\mathrm{PhD}$

Rachel L. Erlich, PhD

Abimbola Farinde, PharmD, MS

Steven R. Feldman, MD, PhD

Stanley E. Ferrell, BSPharm

Erin Ferries, PhD, MPH

Renee R. Fleming, RPh, MBA

Sara Forrester, PharmD, MS

Meg A. Franklin, PharmD, PhD

Jennifer Friderici, MS

Andrew Scott Gaiser, PharmD, MS

Justin Gatwood, PhD, MPH

Margaret E. Gerbasi, PhD

Teresa B. Gibson, PhD

Kristin Gillard, PharmD, PhD

Patrick P. Gleason, PharmD, BCPS

Chester Bernie Good, MD, MPH

J. Kelly Goode, PharmD

Boris Gorsh, PharmD

Jennifer S. Graff, PharmD

Bonnie C. Greenwood, PharmD, BCPS

Matthew C. Grissinger, BSPharm

Paul 0. Gubbins, PharmD

Julian Guest, PhD

Nicole Hahn, PharmD 


\section{Thanks to JMCP Peer Reviewers, 2019 (continued)}

Rachel Halpern, PhD, MPH

Nadia Haque, PharmD, MHSA

James Harnett, PharmD, MS

Joel W. Hay, PhD

Gregory Heindel, PharmD

Lindsey Helm, PharmD

Henry J. Henk, PhD

Inmaculada Hernandez, PharmD, PhD

T. Matthew Hill, PharmD

Connie Ho, PharmD, MBA, CSP

Jane Hoh, PharmD

Natalie S. Hohmann, PharmD

David A. Holdford, PhD

Tove Holm-Larsen, MSc, PhD

Anke-Peggy Holtorf, PhD, MBA

James Hopsicker, RPH, MBA

Monika Hornung, PharmD

Anna Hung, PharmD, PhD, MS

Srihari Jaganathan, MS

Jayani Jayawardhana, $\mathrm{PhD}$

Tara K. Jellison, PharmD, MBA

Yawen Jiang, $\mathrm{PhD}$

Tianze Jiao, PhD

Pravin Shivaji Kamble, BPharm, MTech,

RPh, MS, PhD

Marguerite Kandel, PharmD

Isabell Kang, PharmD

Shellie L. Keast, PharmD, PhD

Marcella Kelley, MHS

Dan Kent, PharmD

Kibum Kim, PhD

Youngran Kim, MPH

Jonathan Kish, PhD, MPH

Stephen Kogut, PhD, MBA, RPh

Mark J. Kohn, PharmD, BCPS

David H. Kreling, PhD

Melanie Kuester, PharmD

Sharon Laforest, PharmD

Kenneth LaPensee, PhD, MPH

Ken A. Lawson, PhD

Emily Leckman-Westin, PhD

Thomas Lee, PharmD
YoonJung Lee, PharmD

Kimberly Lenz, PharmD

Sarah Lerchenfeldt, PharmD

Haitao Li, PharmD, MS

Nanxin Li, PhD, MBA

Joshua N. Liberman, PhD

Tracy Kuo Lin, PhD

F. Ellen Loh, BPharm, PhD, MBA

Gregory A. Low, BPharm, PhD

Kevin Lu, PhD, BPharm

Junjie $\mathrm{Ma}, \mathrm{PhD}$

Elizabeth A. MacLean, PharmD, PhD

Jonathan W. Magness, PharmD

Rohan Mahabaleshwarkar, PhD

Tatiana Makhinova, PhD

Martin Marciniak, RPh, MPP, PhD

T. Joseph Mattingly, PharmD, MBA, PhD

Josephine A. Mauskopf, PhD

Carrie McAdam-Marx, PhD, RPh

Katharine McCarthy, PharmD

Kevin McCloy, PharmD

Jeffrey Scott McCombs, PhD

Blake Tyler McGee, PhD, RN

Trent McLaughlin, PhD

Rohan Medhekar, MPharm, PhD

Darshan Mehta, PhD

Hemalkumar B. Mehta, MS, PhD

Cinzia Metallo, $\mathrm{PhD}$

Shivani Mhatre, $\mathrm{PhD}$

Rick Miller, RPh, MBA, CSP

Manish Mittal, PhD

Penny Mohr, MA

Bushra Muraywid, PharmD

Robert P. Navarro, PharmD

Uche Anadu Ndefo, PharmD

Lynda Nguyen, PharmD

Weiyi Ni, PhD

Heather Nyman, PharmD

Mary 0. Obeng, PharmD

Gary Oderda, PharmD, MPH

Alan Oglesby, MPH

Richard L. Ogletree, Jr., PharmD
Arinze Nkemdirim Okere, PharmD, MS, MBA, BCPS

Abiola 0. Oladapo, PhD

Busuyi Olotu, PhD

Oluwadamilola Onasanya, MD, MPH

Kelsey Ondek, PharmD

Terrence 0'Shea, BSPharm, PharmD

Sonia T. Oskouei, PharmD

Amy K. O'Sullivan, PhD

Gary Owens, MD

Dominic Paolella, PharmD

Shreekant V. Parasuraman, BPharm, PhD

Chanhyun Park, PhD

Jiyeon Joy Park, PharmD

Si Yeon Park, PharmD

Yujin Park, PharmD

Nick C. Patel, PharmD, PhD

Vardhaman Patel, MS, PhD

Dhaval S. Patil, MS

Patti J. Peeples, PhD, MS, RPh

Allison Petrilla, MPH

Matthew K. Pickering, PharmD

Elmor David Pineda, PharmD, MS

Michael Polson, MS, PharmD

Jarred B. Prudencio, PharmD, BC-ADM

Lauren Pusateri-Nilson, PharmD

Bruce Pyenson, FSA, MAAA

Patrick Racsa, MS

Jean Rao, PharmD

Rafia S. Rasu, PhD

Neda Ratanawongsa, MD, MPH

Prabashni Reddy, PharmD

Claiborne (Gene) E. Reeder, RPh, PhD

Lori Reisner, PharmD

Elizabeth Rightmier, PharmD

Kevin Rodondi, PharmD

Joseph S. Ross, MD, MHS

Deanna M. Rowe, PharmD

Elan Rubinstein, PharmD, MPH

Anne Marie Runyan, BA

Michael T. Rupp, PhD, BSPharm, FAPhA

Ruth Ann Ruttenberg, PhD 


\section{JMCP}

\section{Thanks to JMCP Peer Reviewers, 2019 (continued)}

Sheila Ryder, BScPharm, MSc, PGDipStat, $\mathrm{PhD}$ candidate

Prashant Sakharkar, PharmD, MPH Jayashri Sankaranarayanan, MPharm, PhD Vishal H. Saundankar, MS, BPharm Kimberly Saverno, PhD, RPh

Jordan L. Schultz, PharmD

Phil Schwab, PhD

Jordan Sedlacek, PharmD

Michael Serbin, MS, PharmD

Mark S. Shaefer, PharmD

Jason Shafrin, $\mathrm{PhD}$

Ruchit Shah, PhD

Fadia T. Shaya, PhD, MPH

Natalia G. Shcherbakova, PhD

Christina H. Sherrill, PharmD

Anand R. Shewale, MS

Katarzyna Shields, PharmD, MBA

Matthew Silva, PharmD

Jonathan Silver, MD, MS, MBA

Jason C. Simeone, PhD

David Singer, PharmD, MS

Mukul Singhal, PhD, BPharm

Julia F. Slejko, PhD

Jessica Smith, PhD

Judith A. Smith, PharmD, BCOP, CPHQ, FCCP, FISOPP

Margie E. Snyder, PharmD, MPH

Christina A. Spivey, PhD
Joshua J. Spooner, PharmD, MS

Anna Staudt, PharmD

Marilyn Stebbins, PharmD

Craig Stephen Stern, RPh, PharmD, MBA

Karen M. Stockl, PharmD

Samuel Stolpe, PharmD, MPH

Bradley S. Stolshek, PharmD

Wenqing Su, MS

Kent Summers, PhD

Satya Surbhi, PhD, MS

Elizabeth C.S. Swart, BS

Patty Taddei-Allen, PharmD, MBA

Ashley Thomas, PharmD

Ryan Thomas, PharmD

Tara M. Thomas, RPh

Patricia Thornewell, PharmD

Joshua Caleb Toliver, PharmD

Edmond Toy, PhD

Josephine (Nhu) Tran, PharmD, MS

Stephanie Tran, PharmD

Navneet Upadhyay, PhD, MS

Benjamin Y. Urick, PharmD, PhD

Connie A. Valdez, PharmD, MSEd, BCPS

Sumit Verma, MS, BPharm

Rolin L. Wade, RPh, MS

David Wamble, PhD

Junling Wang, PhD

Yan Wang, PhD
Karen Watkins, PharmD, BCOP

Jay Weaver, PharmD, MPH

Albert I. Wertheimer, BS, MBA, PhD

Ria Westergaard, PharmD

Kimberly Westrich, MA

Peter Whittaker, PhD

Daniel Wiederkehr, MPH

Aaron Winn, PhD

Jenifer Wogen, MS

Mary Claire Wohletz, PharmD

Andrew L. Wong, MD

Benjamin Wong, MS

Shui Ling Wong, MS

Joanne $\mathrm{Wu}, \mathrm{MD}, \mathrm{MS}$

Jun $\mathrm{Wu}, \mathrm{PhD}$

Jing Xie, MB, MPH, ScD

Yihua $\mathrm{Xu}, \mathrm{PhD}$

Hongbo Yang, PhD

Winnie M. Yang, PharmD

Xiaoqin Yang, $\mathrm{PhD}$

Kai Yeung, PharmD, PhD

Peinie Young, PharmD

Jeffrey $C$. Yu, AB, MHS

Jing Yuan, BPharm, PhD

Lixian Zhong, $\mathrm{PhD}$

Autumn Zuckerman, PharmD, BCPS, AAHIVP, CSP

Diana Zuckerman, PhD 